\title{
Characterization of Verticillium dahliae and V. tricorpus Isolates from Lettuce and Artichoke
}

Qing-Ming Qin, Department of Plant Pathology, University of California, Davis, c/o United States Agricultural Research Station, Salinas, CA, 93905; Gary E. Vallad, Department of Plant Pathology, University of Florida, Gulf Coast Research and Education Center, Wimauma 33598, and Krishna V. Subbarao, Department of Plant Pathology, University of California, Davis, c/o United States Agricultural Research Station, Salinas

\begin{abstract}
Qin, Q.-M., Vallad, G. E., and Subbarao, K. V. 2008. Characterization of Verticillium dahliae and V. tricorpus isolates from lettuce and artichoke. Plant Dis. 92:69-77.

Verticillium isolates collected from lettuce and artichoke were characterized for morphology, growth and pathogenicity. Several isolates were identified as Verticillium tricorpus based on morphological and cultural characteristics, including the production of dark resting mycelia, chlamydospores, microsclerotia, and yellow to orange pigmentation in culture. Compared with isolates of $V$. dahliae, these isolates also produced microsclerotia and conidia that were significantly larger and exhibited a distinct growth pattern at varying temperatures. Using database sequence information, primers were developed from the internal transcribed spacer region to produce a diagnostic 337-bp product specific to $V$. tricorpus and used to confirm the identification of isolates. Pathogenicity tests indicated that isolates of V. tricorpus were weak pathogens, causing a median disease severity (DS) of $<1$ (0-to-5 scale) on lettuce and artichoke. In contrast, isolates of $V$. dahliae consistently caused severe wilt with a median DS of $>3.5$ on lettuce and 5.0 on artichoke. Although lettuce and artichoke inoculated with isolates of $V$. tricorpus exhibited reduced height and fresh foliar and root weight, the reductions were not statistically significant, unlike in plants inoculated with isolates of $V$. dahliae. Lettuce co-inoculated with isolates of $V$. tricorpus and $V$. dahliae exhibited reduced symptoms of Verticillium wilt and improved growth relative to those inoculated with $V$. dahliae alone. The early introduction of $V$. tricorpus in soil-drench inoculations appeared to provide better relief from subsequent $V$. dahliae inoculation than when the two species were co-inoculated simultaneously using the root-dip method, suggesting competitive exclusion as a plausible mechanism. A spore-polymerase chain reaction assay developed using cultured spores directly as template and primers specific to V. tricorpus confirmed the presence of $V$. tricorpus on inoculated roots. This work demonstrates the potential use of $V$. tricorpus to directly reduce the effect of $V$. dahliae on lettuce and artichoke and, to our knowledge, is the first reported characterization of V. tricorpus isolates collected from lettuce and artichoke.
\end{abstract}

Additional keywords: co-inoculation, colony morphology, cross-protection

The genus Verticillium consists of six phytopathogenic species: Verticillium dahliae Kleb., V. albo-atrum Reinke \& Berthold, V. nigrescens Pethybr., V. nubilum Pethybr., V. tricorpus I. Isaac, and $V$. theobromae (Turconi) E.W. Mason and S. Hughes after the recent revision $(2,23)$. Of the six species, $V$. dahliae and $V$. alboatrum are well-known plant pathogens causing vascular wilt in a broad range of economically important plants, whereas

Corresponding author: K. V. Subbarao

E-mail: kvsubbarao@ucdavis.edu

Q.-M. Qin and G. E. Vallard contributed equally to this work.

Current address of Q.-M. Qin: Department of Plant Pathology and Microbiology, Texas A\&M University.

Accepted for publication 21 August 2007.

doi:10.1094/PDIS-92-1-0069

(C) 2008 The American Phytopathological Society colony, whereas V. tricorpus always produces dark hyphae or dark mycelia and globose to irregular microsclerotia that are much larger in size and are scattered around the colony (9). These differences easily distinguish $V$. tricorpus from other closely related species. However, morphological characteristics are affected by many factors, and loss of resting structures in vitro is common and can be rapid, so that specific isolates may not be easily identifiable (2). Therefore, additional diagnostic methods have been developed to distinguish $V$. tricorpus and $V$. dahliae from other Verticillium spp. $(19,22)$.

Lettuce and artichoke are two important vegetable crops grown year-round in California, with a combined value of over $\$ 1.5$ billion in Monterey County alone. The susceptibility of lettuce, once considered a nonhost of Verticillium species, to V. dahliae was first recognized in 1995 and since has become a serious threat to the lettuce industry in coastal California (28). Dufrenoy first reported $V$. dahliae on artichoke in France in 1927 (23) whereas, in the United States, infection of artichoke was not reported until 1999 (3). In addition to $V$. dahliae, Verticillium isolates morphologically similar to those of $V$. tricorpus also were isolated frequently from diseased lettuce and artichoke in field surveys in recent years. $V$. tricorpus has been reported to be pathogenic to tomato and antirrhinum $(10-13,31)$, as the causal agent of dry rot of stored potato (30), and as a mild pathogen of potato (7). New pathotypes of $V$. tricorpus attacking tomato, eggplant, and potato were found in Tunisia recently (15). Although Verticillium spp. are widely distributed in the agricultural soils of California and affect diverse economically important crops $(3,4,17)$, lettuce and artichoke had not been described previously as hosts of $V$. tricorpus.

The objectives of this study were to better characterize the $V$. tricorpus-like isolates recovered from diseased lettuce and artichoke for morphological and cultural characteristics, and for pathogenicity toward lettuce and artichoke individually and in co-inoculation with $V$. dahliae. A final objective was to diagnose these $V$. tricorpus-like isolates using previously developed primers specific to $V$. tricorpus by polymerase chain reaction (PCR), and to 
develop a rapid method to identify $V$. tricorpus on plant roots.

\section{MATERIALS AND METHODS}

Isolates of Verticillium spp. In all, 19 Verticillium isolates were prepared for these studies (Table 1). The eight isolates of $V$. dahliae and nine isolates of $V$. tricorpus were recovered from Verticillium-wiltsymptomatic lettuce and artichoke grown in coastal California, while the isolate of $V$. albo-atrum and the long-spored isolate of $V$. dahliae var. longisporum (also referred to as $V$. longisporum) were recovered from alfalfa and horseradish, respectively. All the isolates were single spored and maintained on potato dextrose agar (PDA) or as a spore suspension as previously described (24).

Morphology and colony growth of isolates. All cultures were grown for 4 weeks at $25^{\circ} \mathrm{C}$ prior to morphological characterization. The lengths and widths of 40 randomly chosen conidia, microsclerotia, and chlamydospores of the eight isolates of $V$. dahliae and nine isolates of $V$. tricorpus were measured under a microscope. During incubation, potential changes in colony morphology were recorded twice a week.

Two experiments were conducted to compare the response of Verticillium isolates to temperature. In the first experiment, the growth response of the 17 isolates from lettuce and artichoke was tested at $5,10,15,20,25,30$, and $35^{\circ} \mathrm{C}$ in the dark. Test cultures were initiated with a 4.5-mm-diameter mycelial plug taken from the leading edge of a colony previously grown for 2 weeks in the dark at $25^{\circ} \mathrm{C}$. The diameters of colonies were measured after 2 weeks of incubation. For the second experiment, a smaller, more diverse collec- tion of six isolates was tested (Ms.103, Ar.136, Ls.16, Ls.17, Ls.183, and Cs.225) for their growth response at 15, 20, 25, and $30^{\circ} \mathrm{C}$. The diameters of colonies were then measured every 2 days. In both experiments, four plates of each isolate were incubated at each temperature and repeated once $(n=2)$.

Isolate identification by PCR. Two $V$. tricorpus-specific primers from the internal transcribed spacer (ITS) region, VtITS1: 5'-CGCCGGTACATCAGTCTC$3^{\prime}$ and VtITS2: 5'-ACTCCGATGCGA GCGAA-3', developed previously (22) were used to further differentiate isolates of $V$. tricorpus from other phytopathogenic Verticillium spp. The primers were supplied by Sigma-Genosys (The Woodlands, TX). Amplified genomic DNA was extracted from frozen mycelia following the method described by Al-Samarrai and Schmid (1). PCR amplifications were performed with a PTC-200 thermocyler (MJ Research, Waltham, MA) in a total of $25 \mu \mathrm{l}$ of mixture as previously described (24). Samples were overlaid with a drop of mineral oil and PCR reactions were run under an initial denaturation at $95^{\circ} \mathrm{C}$ for $5 \mathrm{~min}$; 35 cycles of denaturation at $95^{\circ} \mathrm{C}$ for 1 min, annealing at $60^{\circ} \mathrm{C}$ for $50 \mathrm{~s}$, and extension at $72^{\circ} \mathrm{C}$ for $1 \mathrm{~min}$; followed by a final extension at $72^{\circ} \mathrm{C}$ for $5 \mathrm{~min}$, and stored at $4^{\circ} \mathrm{C}$ until used. The PCR products were analyzed on a $1.0 \%$ agarose gel in $0.5 \times$ Tris-acetate-EDTA buffer (25).

Pathogenicity of isolates and sporePCR. To evaluate the pathogenicity of different isolates either singly or in combination, lettuce and artichoke seed were sown into 200-well seedling trays filled with an autoclaved sand:potting soil mix (3:1, vol/vol). Seedlings were maintained

Table 1. Isolates of Verticillium used in the study along with their host and geographic origin

\begin{tabular}{|c|c|c|c|}
\hline Species, isolate & Originating host & $\begin{array}{c}\text { Geographical } \\
\text { location }\end{array}$ & $\begin{array}{c}\text { Year } \\
\text { isolated }\end{array}$ \\
\hline \multicolumn{4}{|c|}{ Verticillium dahliae } \\
\hline Cs.80 & Artichoke (Cynara scolymus L.) & California & 1991 \\
\hline C.312 & Artichoke (C. scolymus L.) & California & 1999 \\
\hline Cs.413 & Artichoke (C. scolymus L.) & California & 2000 \\
\hline Cs.423 & Artichoke (C. scolymus L.) & California & 2001 \\
\hline Ls.1 & Lettuce (Lactuca sativa L.) & California & 1995 \\
\hline Ls.14 & Lettuce (L. sativa $\mathrm{L}$.) & California & 1996 \\
\hline Ls.16 & Lettuce (L. sativa L.) & California & 1996 \\
\hline Ls.17 & Lettuce (L. sativa L.) & California & 1996 \\
\hline \multicolumn{4}{|l|}{ V. tricorpus } \\
\hline Cs. 225 & Artichoke (C. scolymus L.) & California & 1999 \\
\hline Cs.234 & Artichoke (C. scolymus L.) & California & 1999 \\
\hline Cs.236 & Artichoke (C. scolymus L.) & California & 1999 \\
\hline Cs. 456 & Artichoke (C. scolymus L.) & California & 1999 \\
\hline Ls.183 & Lettuce (L. sativa L.) & California & 1997 \\
\hline Ls.432 & Lettuce (L. sativa $\mathrm{L}$.) & California & 2001 \\
\hline Ls.441 & Lettuce (L. sativa L.) & California & 2001 \\
\hline Ls.442 & Lettuce (L. sativa $\mathrm{L}$.) & California & 2001 \\
\hline Ls. 443 & Lettuce (L. sativa L.) & California & 2001 \\
\hline \multicolumn{4}{|c|}{ V. dahliae var. longisporum } \\
\hline Ar. $136^{\mathrm{a}}$ & Horseradish (Armoracia rusticana) & Illinois & 1989 \\
\hline \multicolumn{4}{|l|}{ V. albo-atrum } \\
\hline Ms. $103^{\mathrm{b}}$ & Alfalfa (Medicago sativus L.) & Pennsylvania & 1986 \\
\hline
\end{tabular}

on benches in a greenhouse (for experiments using root dip inoculation) as previously described (24) or in a growth chamber (for experiments using soil-drench inoculation) at $25^{\circ} \mathrm{C}$ and a 12 -h photoperiod. For experiments using the root-dip inoculation method, 10 4-week-old seedlings with intact roots were inoculated with a conidial suspension of approximately 1.0 $\times 10^{7}$ conidia $/ \mathrm{ml}$. Ten seedlings of both lettuce and artichoke were dipped in each conidial suspension treatment $(50 \mathrm{ml})$ for at least $30 \mathrm{~min}$. Seedlings then were transplanted individually into 0.5 -liter foaminsulated cups containing a pasteurized sand:potting soil mix $(3: 1, \mathrm{vol} / \mathrm{vol})$. Seedlings dipped in sterile water were treated as controls.

For soil-drench inoculation, 4-week-old seedlings were inoculated by adding 1.5 $\mathrm{ml}$ of conidial suspension $\left(2.0 \times 10^{6} \mathrm{co}-\right.$ nidia/ml) to each seedling tray well. For mixed inoculation treatments, $V$. tricorpus isolates were applied a week prior to the addition of $V$. dahliae isolates; each isolate was applied in equal measure. A separate set of seedlings were drenched with sterile water for controls. Fifteen seedlings were inoculated with each isolate or combination of isolates. Seedlings were similarly transplanted into 0.5-liter foam-insulated cups containing a pasteurized sand:potting soil mix (3:1 vol/vol). In each experiment, plants were arranged in a randomized block design on greenhouse benches. Each treatment had three blocks, and each block contained three to five plants for each treatment. The lettuce and artichoke experiments using the root dip inoculation method were repeated once $(n=2)$ and the experiment using the soil-drench inoculation method was repeated three times $(n=4)$.

All plants were gently uprooted 7 to 10 weeks after transplanting, washed free of soil, and rated for disease severity by longitudinally cutting from the crown through the main taproot of each plant as previously described (24). Plant height and width were measured prior to uprooting. The fresh foliar and root weight were determined using a portable electric scale. For reisolation, plant roots were washed with tap water for 1 to $2 \mathrm{~min}$ and dried on sterile paper towel, and 1- to $2-\mathrm{cm}$ sections of excised root tissue (six sections per petri dish) were placed on a modified NP-10 medium (16) and incubated in the dark at 18 to $25^{\circ} \mathrm{C}$ for 10 days. After 10 days of incubation, morphological characteristics such as the presence of microsclerotia, pigmentation, and melanized hyphae were examined under both stereo and compound microscopes (BX60 and SZX; Olympus, Japan), and the spores also were identified directly via the spore-PCR method using primers specific to $V$. tricorpus.

The spore-PCR assay was performed directly on conidia collected from colonies growing around root-tissue-sections previ- 
ously placed on modified NP-10 medium. Conidia were collected using a rubber policeman to gently dislodge conidia around the plated root tissue in $1 \mathrm{ml}$ of sterile water. The suspension was transferred to a $1.5-\mathrm{ml}$ microcentrifuge tube with a pipette and brought to a $1.5-\mathrm{ml}$ total volume with sterile water. The suspension was vortexed repeatedly and centrifuged at $2,300 \times g$ for $1 \mathrm{~min}$, discarding the supernatant in between until the final spore concentration achieved was $1.0 \times 10^{8}$ conidia $/ \mathrm{ml}$. The samples were stored at $-20^{\circ} \mathrm{C}$ until use in PCR assays. Spore-PCR amplification was conducted in a total of $25 \mu$ of reaction mixture containing approximately 1.5 to $2.0 \mu \mathrm{l}$ of spore suspension ( 1.5 to $2.0 \times 10^{5}$ conidia) as template, $2.5 \mu$ l of $10 \times$ PCR buffer, $2.5 \mu \mathrm{l}$ of $25 \mathrm{mM}$ $\mathrm{Mg}^{2+}, 0.2 \mathrm{mM}$ each dNTP, $0.2 \mu \mathrm{M}$ each primer, and 1.25 units of $T a q$ polymerase (Promega Corp., Madison, WI). Samples were overlaid with a drop of mineral oil and subjected to the PCR conditions described above. PCR products were analyzed on a $1.0 \%$ agarose gel.

Data analysis. Mean lengths and widths of conidia and microsclerotia of different $V$. dahliae and $V$. tricorpus isolates were computed and significant differences were determined using paired $t$ tests. Initial analyses of disease severity and biomass data were consistent across repeated experiments; therefore, data were pooled and analyzed according to crop. For disease severity, the combined dataset was analyzed using a nonparametric procedure for the analysis of ordinal data in one-way (artichoke) and two-way (lettuce) factorial experiments $(5,26)$. The overall effect of $V$. dahliae isolates, $V$. tricorpus isolates, and mixtures of the two on the severity of disease was analyzed by the analysis of vari- ance type statistic of ranked data using the PROC Mixed procedure in SAS (version 9.1; SAS Institute Inc., Cary, NC) to generate relative marginal effects (RME), and the LD_CI macro to generate $95 \%$ confidence intervals $(5,26)$. Replications of experiment and blocks within experiments were treated as random effects in the analysis. Linear contrasts were performed to test specific interactions within models. Data for plant height and biomass (fresh foliar and root weight) from pathogenicity experiments were similarly analyzed for a two-way analysis of variance using the PROC Mixed procedure of SAS (version 9.1; SAS Institute Inc.) with differences between least significant means tested using Tukey-Kramer pairwise comparisons.

\section{RESULTS}

Morphological characterization. During the 4-week incubation, colonies of eight isolates of $V$. dahliae (Ls.1, Ls.14, Ls.16, Ls.17, Cs.80, Cs.312, Cs.413, and Cs.423) changed from white to black as they melanized, while colonies of isolates Ls.183, Ls.442, Cs.225, Cs.234, Cs.236, and Cs.456 produced a yellow-orange pigmentation in the first 2 to 3 weeks, before turning black. However, the yellow-orange pigmentation was still apparent around the edge of the growing colony after 4 weeks of incubation. Colonies of isolates Ls.432, Ls.441, and Ls.443 changed from white to black only (Table 2).

The conidial size (length by width) of the eight isolates of $V$. dahliae ranged from 3.2 to 9.0 by 1.4 to $3.2 \mu \mathrm{m}$, with an average size of 5.0 by $2.2 \mu \mathrm{m}$. The conidial sizes of the other Verticillium isolates ranged from 3.5 to 11.0 by 1.8 to $3.8 \mu \mathrm{m}$, with an average of 6.2 by $2.6 \mu \mathrm{m}$ (Table 2 ). The differences in conidial lengths and widths between these two groups of isolates were significant (Table 3 ). The size of microsclerotia of the eight isolates of $V$. dahliae ranged from 16.2 to 150.0 by 12.5 to $105.0 \mu \mathrm{m}$, with a mean size of 59.7 by $37.2 \mu \mathrm{m}$. Microsclerotia of the other Verticillium isolates ranged from 15.0 to 230.0 by 10.0 to $150.0 \mu \mathrm{m}$, with a mean size of 90.9 by $57.7 \mu \mathrm{m}$ (Table 2). Again, the differences in mean microsclerotial lengths and widths between these two groups of isolates were significant (Table 3). The chlamydospores of Ls.183, Ls.432, Ls.441, Ls.442, Ls.443, Cs.225, Cs.234, Cs.236, and Cs.456 ranged from 4.5 to 13.0 by 3.5 to $9.0 \mu \mathrm{m}$, with a mean of 7.9 by $6.1 \mu \mathrm{m}$. Chlamydospores were never observed among the eight isolates of $V$. dahliae (Table 2 and data not shown). In addition, Ls.183, Ls.432, Ls.441, Ls.442, Ls.443,

Table 3. Comparison of the differences in lengths and widths of conidia and microsclerotia between isolates of Verticillium dahliae and V. tricorpus ${ }^{\mathrm{a}}$

\begin{tabular}{lcccc}
\hline Comparison $^{\mathbf{b}}$ & SD & $\boldsymbol{t}$ Statistic & df & Significance \\
\hline Mean conidial length & 0.70 & 0.00003 & 16 & $P<0.001$ \\
Mean conidial width & 0.24 & 0.00001 & 16 & $P<0.001$ \\
Mean microsclerotial length & 23.46 & 0.00373 & 16 & $P<0.01$ \\
Mean microsclerotial width & 17.02 & 0.01019 & 16 & $P<0.05$ \\
\hline
\end{tabular}

a $\mathrm{SD}=$ standard deviation and $\mathrm{df}=$ degrees of freedom.

${ }^{\mathrm{b}}$ V. dahliae versus V. tricorpus isolates.

Table 2. Comparison of morphological characteristics of Verticillium dahliae and V. tricorpus isolates from lettuce and artichoke ${ }^{\mathrm{a}}$

\begin{tabular}{|c|c|c|c|c|c|c|c|}
\hline \multirow[b]{2}{*}{ Isolates } & \multicolumn{2}{|c|}{ Spore } & \multicolumn{2}{|c|}{ Microsclerotia } & \multicolumn{2}{|c|}{ Chlamydospore } & \multirow[b]{2}{*}{ Color change } \\
\hline & Length $(\mu \mathrm{m})$ & Width $(\mu \mathrm{m})$ & Length $(\mu \mathrm{m})$ & Width $(\mu \mathrm{m})$ & Length $(\mu \mathrm{m})$ & Width $(\mu \mathrm{m})$ & \\
\hline \multicolumn{8}{|l|}{ V. dahliae } \\
\hline Ls.1 & $5.3(1.1)$ & $2.2(0.3)$ & $62.1(27.0)$ & $35.0(12.3)$ & Absent & Absent & White to black \\
\hline Ls.14 & $5.4(1.1)$ & $2.2(0.3)$ & $57.5(18.4)$ & $40.5(8.8)$ & Absent & Absent & White to black \\
\hline Ls.16 & $5.5(0.9)$ & $2.3(0.4)$ & $57.6(20.6)$ & $41.1(14.3)$ & Absent & Absent & White to black \\
\hline Ls.17 & $5.0(0.9)$ & $2.4(0.4)$ & $53.8(24.5)$ & $29.8(10.8)$ & Absent & Absent & White to black \\
\hline Cs. 80 & $4.3(0.7)$ & $2.2(0.4)$ & $64.9(27.3)$ & $40.6(16.5)$ & Absent & Absent & White to black \\
\hline Cs. 312 & $4.5(0.7)$ & $2.1(0.4)$ & $57.3(18.5)$ & $37.0(11.3)$ & Absent & Absent & White to black \\
\hline Cs. 413 & $5.0(1.2)$ & $2.2(0.5)$ & 73.7 (31.3) & $41.2(18.1)$ & Absent & Absent & White to black \\
\hline Cs. 423 & $5.0(0.6)$ & $2.1(0.4)$ & $52.4(22.4)$ & $32.1(8.1)$ & Absent & Absent & White to black \\
\hline Mean & 5.0 & 2.2 & 59.7 & 37.2 & $\ldots$ & $\ldots$ & $\ldots$ \\
\hline \multicolumn{8}{|l|}{ V. tricorpus } \\
\hline Ls. 183 & $6.5(1.8)$ & $2.7(0.3)$ & $93.9(36.7)$ & $58.2(23.3)$ & $7.5(1.5)$ & $6.0(1.1)$ & Yellow to black \\
\hline Ls. 432 & $6.3(1.1)$ & $2.6(0.4)$ & $58.6(35.4)$ & $37.8(21.2)$ & $8.2(1.4)$ & $6.5(0.8)$ & White to black \\
\hline Ls.441 & $6.2(1.1)$ & $2.8(0.4)$ & $85.8(46.5)$ & $51.9(19.1)$ & $8.3(1.7)$ & $6.2(1.0)$ & White to black \\
\hline Ls.442 & $6.3(1.4)$ & $2.6(0.4)$ & $103.6(47.3)$ & $71.2(31.9)$ & $8.5(1.7)$ & $6.0(1.0)$ & Yellow to black \\
\hline Ls. 443 & $6.2(1.3)$ & $2.7(0.4)$ & $82.6(36.9)$ & 49.4 (17.3) & $7.2(0.9)$ & $5.7(0.8)$ & White to black \\
\hline Cs. 225 & $5.7(1.1)$ & $2.3(0.3)$ & $102.4(46.5)$ & $71.0(28.1)$ & $8.1(1.2)$ & $6.2(0.9)$ & Yellow to black \\
\hline Cs.234-2 & $6.3(1.0)$ & $2.7(0.5)$ & $61.2(29.0)$ & $31.1(10.4)$ & $7.7(1.5)$ & $6.2(1.0)$ & Yellow to black \\
\hline Cs.236 & $5.8(1.4)$ & $2.5(0.4)$ & $94.2(37.0)$ & $56.8(17.1)$ & $8.1(1.1)$ & $6.1(1.0)$ & Yellow to black \\
\hline Cs. 456 & $6.4(1.4)$ & $2.6(0.4)$ & $136.1(43.5)$ & $91.6(20.1)$ & $7.6(1.9)$ & $6.0(1.3)$ & Yellow to black \\
\hline Mean & 6.2 & 2.6 & 90.9 & 57.7 & 7.9 & 6.1 & $\ldots$ \\
\hline
\end{tabular}

a Average size of 40 conidia, microsclerotia, chlamydospores and standard error of different measurements in parentheses.

${ }^{\mathrm{b}}$ Change of colony color. 
Cs.225, Cs.234, Cs.236, and Cs.456 produced dark resting mycelia which were absent in the isolates of $V$. dahliae (data not shown). The above morphological characteristics for isolates Ls.183, Ls.432, Ls.441, Ls.442, Ls.443, Cs.225, Cs.234, Cs.236, and Cs.456 were distinct from those described for $V$. dahliae or $V$. albo-atrum, but typical of $V$. tricorpus $(11,13)$ and hereafter were referred to as such.

Response of isolates to temperature. All isolates from lettuce and artichoke grew at temperatures between 5 and $30^{\circ} \mathrm{C}$ (Fig. 1). The maximum growth of the eight isolates of $V$. dahliae occurred at $25^{\circ} \mathrm{C}$,

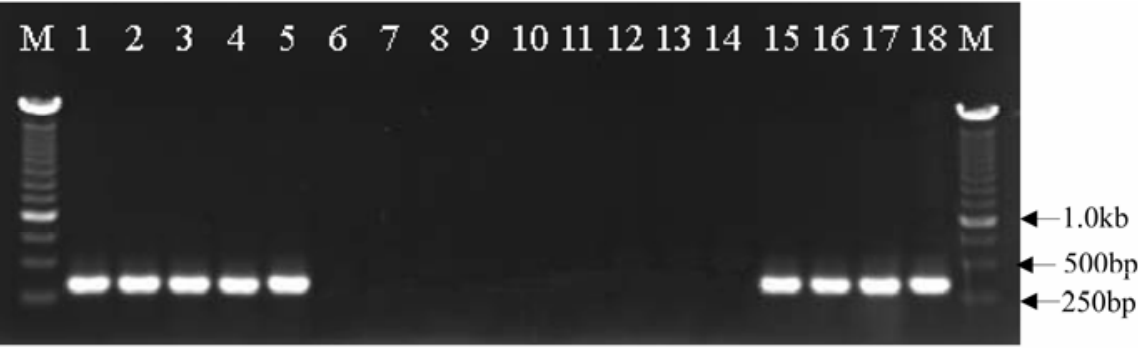

Fig. 2. Polymerase chain reaction identification of Verticillium tricorpus isolates with $V$. tricorpus-specific primers. Lanes 1-5 are PCR products, from V. tricorpus isolates Ls.183, Ls.432, Ls.441, and Ls.442 and Ls. 443 from lettuce; lanes 6-9 are from lettuce $V$. dahliae isolates Ls.1, Ls.14, Ls.16, and Ls.17; lane 10 is from control (sterile water); lanes 11-14 are from $V$. dahliae isolates Cs.80, Cs.312, CS.413, and Cs.423 from artichoke; and lanes 15-18 are from V. tricorpus isolates Cs.225, Cs.234, Cs.236, and Cs.456 from artichoke. Each lane was loaded with $6 \mu \mathrm{l}$ of PCR product. M indicates 250-bp DNA ladder (Invitrogen).

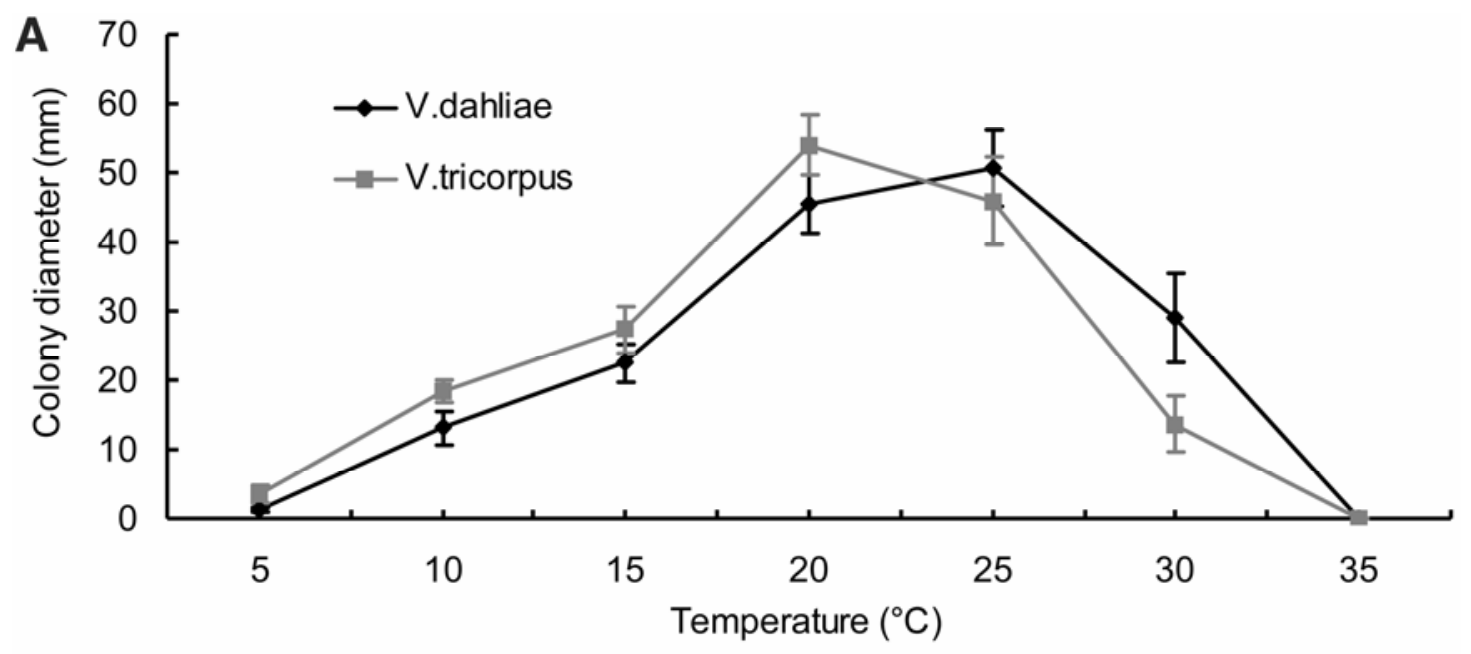

B
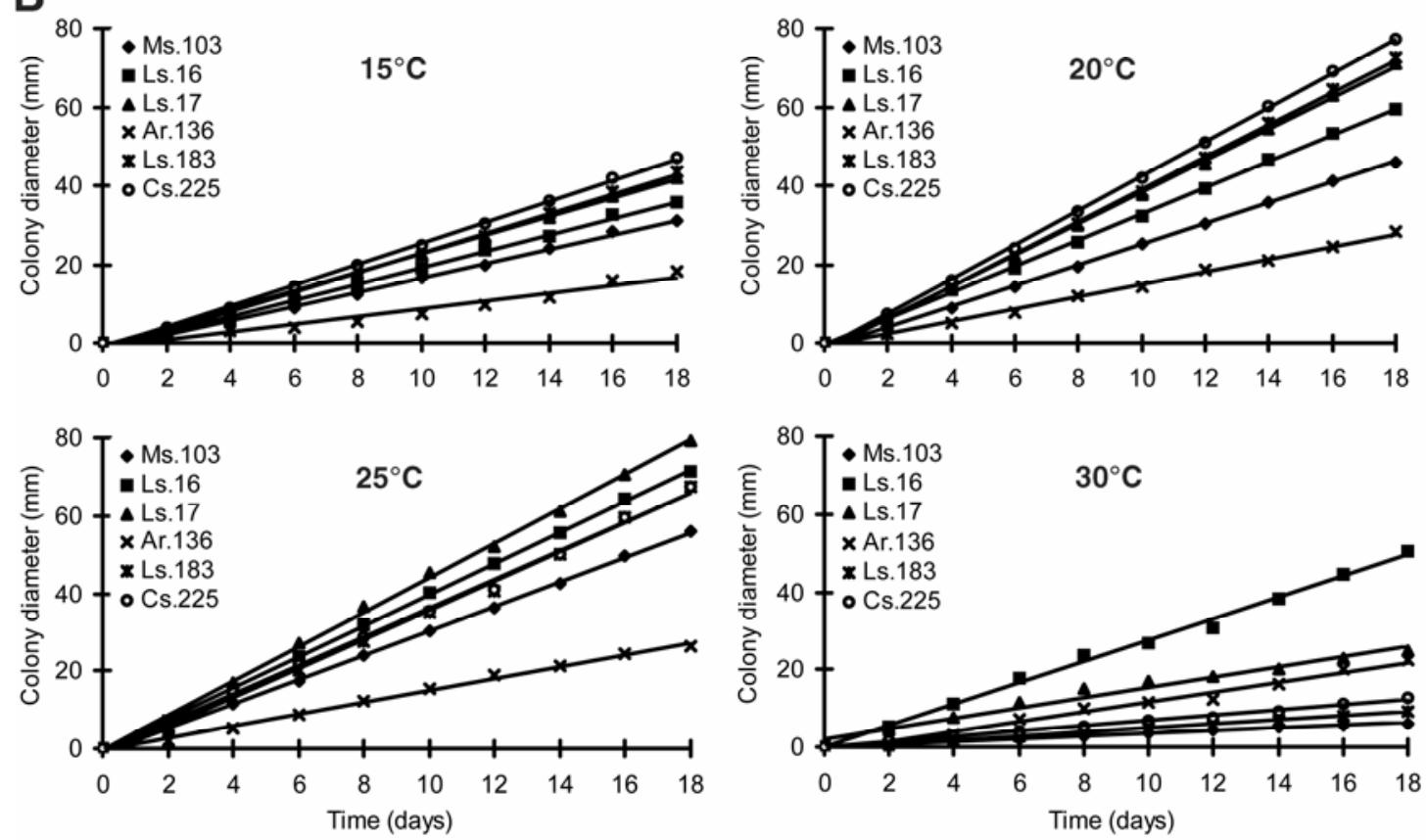

Fig. 1. Effect of temperature on the rate of growth of Verticillium tricorpus relative to other Verticillium spp. under controlled conditions. A, Comparison of the average rate of growth of nine isolates of V. dahliae (Ls.1, Ls.7, Ls.14, Ls.16, Ls.17, Cs.80, Cs.312, Cs.413, and Cs.423) versus the average of nine isolates of V. tricorpus (Ls.183, Ls.432, Ls.441, Ls.442, Ls.443, Cs.225, Cs.234, Cs.236, and Cs.456) following 2 weeks of incubation at various temperatures. B, Average growth of V. tricorpus isolates Ls.183 and Cs.225, V. dahliae isolates Ls.16 and Ls.17, V. dahliae var. longisporum isolate Ar.136, and V. alboatrum isolate Ms. 103 up to 18 days at $15,20,25$, and $30^{\circ} \mathrm{C}$. Growth was measured by the increased diameter (mm) of colonies on potato dextrose agar medium over time. Data represent the means of growth ( \pm the standard deviation in B) from two independent experiments. Isolate prefixes refers to the crop of origin: "Ar" for horseradish, "Cs." for artichoke, "Ls" for lettuce, and "Ms." for alfalfa 
whereas that of the nine isolates of $V$. tricorpus occurred at $20^{\circ} \mathrm{C}$ (Fig. 1). The rate of growth for most Verticillium isolates was inhibited at $30^{\circ} \mathrm{C}$, especially the nine isolates of $V$. tricorpus (Ls.183, Ls.432, Ls.441, Ls.442, Ls.443, Cs.225, Cs.234, Cs.236, and Cs.456) and the single V. alboatrum isolate, Ms.103 (Fig. 1). Of all the isolates tested, isolate Ls.16 ( $V$. dahliae) was the most tolerant to the elevated temperature of $30^{\circ} \mathrm{C}$ (Fig. 1). The growth of all isolates was inhibited at $35^{\circ} \mathrm{C}$ (Fig. 1). After 15 days of incubation at $35^{\circ} \mathrm{C}$, colonies with older mycelia or resting structures still could be revived when transferred to $20^{\circ} \mathrm{C}$, whereas younger colonies lacking older mycelia or resting structures could not be revived. None of the isolates survived after 90 days incubation at $35^{\circ} \mathrm{C}$ (data not shown).

PCR identification. Using the $V$. tricorpus-specific primers VtITS1 and VtITS2, a diagnostic 337-bp fragment was amplified from the $V$. tricorpus isolates Ls.183, Ls.432, Ls.441, Ls.442, and Ls.443 from lettuce and isolates Cs.225, Cs.234, Cs.236, and Cs.456 from artichoke (Fig. 2). No product was amplified from any isolate of $V$. dahliae, $V$. dahliae var. longisporum, or V. albo-atrum (Fig. 2 and data not shown). These results corroborated the previous morphological characterization and confirmed that isolates Ls.183, Ls.432, Ls.441, Ls.442, Ls.443, Cs.225, Cs.234, Cs.236, and Cs.456 were indeed V. tricorpus.

Pathogenicity on lettuce. Regardless of the inoculation method, the overall effect of $V$. dahliae isolate on disease severity was highly significant $(P<0.0001$ for both inoculation methods; Table 4). Median disease severity scores ranged from 3.8 to 4.6 for lettuce inoculated with either $V$. dahliae isolate Ls.16 or Ls.17 alone (Table 5). Although no difference in disease severity between plants inoculated with either Ls.16 or Ls.17 alone was apparent $(P$ $=0.4962)$ via the root-dip method, a significant difference $(P<0.0001)$ was observed with the soil-drench method, with
Table 5. Median (Med), mean ranking $(R)$, and relative marginal effect (RME) calculated for the severity of Verticillium wilt on lettuce inoculated with isolates of Verticillium dahliae and V. tricorpus ${ }^{\mathrm{a}}$

\begin{tabular}{lclll}
\hline${\text { V. } \text { dahliae }^{\mathbf{b}}}^{\text {Root-dip inoculation }}$ & V. tricorpus $^{\mathbf{b}}$ & Med & $\boldsymbol{R}^{\mathbf{c}}$ & RME $^{\mathbf{d}}$ \\
$\ldots$ & $\ldots$ & & & \\
$\ldots$ & $\ldots$ & 0 & 15.5 & $0.14(0.10-0.20)$ \\
$\ldots$ & Ls.183 & 0.5 & 29.2 & $0.27(0.20-0.35)$ \\
$\ldots$ & Ls.432 & 0 & 17.8 & $0.16(0.10-0.27)$ \\
$\ldots$ & Ls.441 & 0.7 & 30.1 & $0.27(0.17-0.43)$ \\
$\ldots$ & Ls.442 & 0.6 & 29.5 & $0.27(0.18-0.38)$ \\
Ls.16 & Ls.443 & 0.5 & 26.3 & $0.24(0.17-0.33)$ \\
Ls.16 & $\ldots$ & 4 & 87.7 & $0.81(0.69-0.88)$ \\
Ls.16 & Ls.183 & 3.5 & 79.5 & $0.73(0.60-0.83)$ \\
Ls.16 & Ls.432 & 4.5 & 84 & $0.77(0.55-0.90)$ \\
Ls.16 & Ls.441 & 3.5 & 77.2 & $0.71(0.59-0.81)$ \\
Ls.16 & Ls.442 & 4.6 & 88.4 & $0.81(0.66-0.90)$ \\
Ls.17 & Ls.443 & 3.5 & 64.8 & $0.60(0.38-0.78)$ \\
Ls.17 & $\ldots$ & 3.8 & 80.8 & $0.74(0.57-0.86)$ \\
Ls.17 & Ls.183 & 3.6 & 68.2 & $0.63(0.39-0.81)$ \\
Ls.17 & Ls.432 & 1.9 & 53.6 & $0.49(0.30-0.68)$ \\
Ls.17 & Ls.441 & 2 & 54 & $0.50(0.38-0.61)$ \\
Ls.17 & Ls.442 & 2.3 & 58.5 & $0.54(0.34-0.72)$ \\
Soil-drench inoculation & Ls.443 & 1 & 36.1 & $0.33(0.18-0.53)$ \\
$\ldots$ & & & & \\
$\ldots$ & $\ldots$ & 0 & 7 & $0.06(0.06-0.09)$ \\
$\ldots$ & Ls.432 & 0.6 & 24.4 & $0.22(0.19-0.26)$ \\
Ls.16 & Ls.443 & 0.8 & 27.7 & $0.25(0.21-0.31)$ \\
Ls.16 & $\ldots$ & 4.6 & 98.1 & $0.90(0.86-0.93)$ \\
Ls.16 & Ls.432 & 3.1 & 74 & $0.68(0.57-0.77)$ \\
Ls.17 & Ls.443 & 2.5 & 64.1 & $0.59(0.52-0.66)$ \\
Ls.17 & $\ldots$ & 3.8 & 83.8 & $0.77(0.71-0.82)$ \\
Ls.17 & Ls.432 & 1.8 & 54.8 & $0.50(0.41-0.59)$ \\
\hline & Ls.443 & 2 & 56.6 & $0.52(0.45-0.59)$ \\
\hline
\end{tabular}

${ }^{a}$ Results from two and four independent experiments using the root-dip and soil-drench inoculation method, respectively. For experiments using the root-dip inoculation method, the intact roots of 4week-old seedlings were dipped in a suspension of approximately $1.0 \times 10^{7}$ conidia/ml from individual isolates or mixed suspension of each $V$. dahliae and V. tricorpus isolate was mixed in equal measure. For soil-drench inoculation, a $1.5-\mathrm{ml}$ suspension of approximately $2.0 \times 10^{6}$ conidia/ml was applied to the soil of individual 4-week-old seedlings grown in seedling trays. For mixed inoculation treatments, $V$. tricorpus isolates were applied a week prior to the addition of $V$. dahliae isolates in equal measure. Sterile water was used in both methods as a substitute for one or both isolates. Seedlings were transplanted individually into 0.5 -liter foam-insulated cups containing a pasteurized soil mix.

${ }^{\mathrm{b}}$ Seedlings were treated with a single isolate of either V. dahliae or V. tricorpus, or an isolate of each that originally was collected from field-grown lettuce (Lactuca sativa [Ls]); ... designates control, those plants treated with sterile water as a substitute for one or both isolates.

${ }^{\mathrm{c}}$ Mean rankings of Verticillium wilt severity for each isolate treatment. Disease severity was rated using an ordinal scale of 0 to 5 , in which $0=$ no vascular discoloration in the taproot, $1=1$ to $25 \%, 2$ $=26$ to $50 \%, 3=51$ to $75 \%$, and $4=76$ to $100 \%$ of vascular tissues in the taproot exhibited discoloration in the absence of foliar symptoms, and $5=100 \%$ of vascular tissues exhibited discoloration in the taproot and the presence of foliar symptoms typical of Verticillium wilt.

${ }^{\mathrm{d}} \mathrm{RME}=[(R-0.5) / \mathrm{N}] ; \mathrm{N}=$ total experimental units in the analysis $(\mathrm{N}=120$ and 108 in root-dip and soil-drench inoculation methods, respectively). The $95 \%$ confidence intervals are in parentheses.

Table 4. Statistical analyses of variance (ANOVA) based on the effect of individual isolates of Verticillium dahliae and V. tricorpus or mixtures of isolates on the ranked means of Verticillium wilt severity and the means of lettuce growth measurements ${ }^{\mathrm{a}}$

\begin{tabular}{|c|c|c|c|c|c|c|c|c|c|c|c|c|c|c|c|c|}
\hline \multirow[b]{3}{*}{ Isolate } & \multicolumn{8}{|c|}{ ANOVA-type statistic (ATS), disease severity effect ${ }^{b}$} & \multicolumn{8}{|c|}{ ANOVA $F$ statistic $(F)$, lettuce growth effect ${ }^{\mathrm{c}}$} \\
\hline & \multicolumn{4}{|c|}{ Root-dip $(n=2)$} & \multicolumn{4}{|c|}{ Soil-drench $(n=4)$} & \multicolumn{4}{|c|}{ Root weight $(n=4)$} & \multicolumn{4}{|c|}{ Foliar weight $(n=4)$} \\
\hline & $\mathbf{d f}_{\mathrm{Num}}$ & $\mathbf{d f}_{\text {Dem }}$ & ATS & $P$ value & $\mathbf{d f}_{\mathrm{Num}}$ & $\mathbf{d f}_{\text {Dem }}$ & ATS & $P$ value & $\mathbf{d f}_{\mathrm{Num}}$ & $\mathbf{d f}_{\text {Dem }}$ & $\boldsymbol{F}$ & $P$ value & $\mathbf{d f}_{\mathrm{Num}}$ & $\mathbf{d f}_{\text {Dem }}$ & $\boldsymbol{F}$ & $P$ value \\
\hline V. dahliae (D) & 1.77 & 51 & 68.63 & $<0.0001$ & 1.82 & 49.3 & 188 & $<0.0001$ & 2 & 88 & 10.64 & $<0.0001$ & 2 & 88 & 138.7 & $<0.0001$ \\
\hline V. tricorpus $(\mathrm{T})$ & 4.68 & 51 & 2.09 & 0.0853 & 1.75 & 49.3 & 10.68 & 0.0003 & 2 & 88 & 0.61 & 0.5457 & 2 & 88 & 0.54 & 0.583 \\
\hline $\mathrm{D} \times \mathrm{T}$ & 7.87 & $\infty$ & 1.47 & 0.1629 & 3.07 & $\infty$ & 17.36 & $<0.0001$ & 4 & 88 & 1.31 & 0.2723 & 4 & 88 & 3.86 & 0.0062 \\
\hline
\end{tabular}

${ }^{a}$ Abbreviations: $n=$ the number of independent experiments in each analysis, $\mathrm{df}_{\mathrm{Num}}=$ numerator degrees of freedom, and $\mathrm{df}_{\text {Dem }}=\mathrm{denominator}_{\text {degrees of }}$ freedom.

${ }^{\mathrm{b}}$ In the root-dip inoculation method, the roots of 4-week-old seedlings were dipped in a suspension of approximately $1.0 \times 10^{7}$ conidia/ml for $30 \mathrm{~min}$. Mixed inoculation treatments used a suspension of $1.0 \times 10^{7}$ conidia/ml of each V. dahliae and V. tricorpus isolate in equal measure. In the soil-drench inoculation method, 4-week-old seedlings were inoculated by adding a 1.5 - $\mathrm{ml}$ suspension of approximately $2.0 \times 10^{6} \mathrm{conidia} / \mathrm{ml}$ to each seedling tray well. For mixed inoculation treatments, an isolate of $V$. tricorpus was applied 2 week prior to the addition of the isolate of $V$. dahliae; each isolate was applied in equal measure. For both inoculation methods, seedlings were transplanted into 0.5-liter foam-insulated cups containing a pasteurized sand:potting soil mix (3:1, $\mathrm{vol} / \mathrm{vol})$, and isolates were substituted with sterile water in controls.

${ }^{c}$ The effect of $V$. dahliae and V. tricorpus isolates on lettuce growth, in terms of fresh foliar and fresh root weight, was analyzed from experiments using the soil-drench inoculation method. 
Ls.17 exhibiting a $17 \%$ reduction in median disease severity (Table 5). The effect of $V$. dahliae isolate on fresh root and foliar weight also was highly significant $(P$ $<0.0001$ for both) for the soil-drenchinoculated lettuce plants, with plants exhibiting an average reduction of 54.7 and $21.3 \%$ for fresh foliar and root weight, respectively, relative to the noninoculated controls (Fig. 3).

The overall effect of $V$. tricorpus isolate on disease severity was highly significant $(P=0.0003)$ for the soil-drench inoculation method but less significant $(P=$ 0.0853 ) with the root-dip inoculation method (Table 4). Regardless, the severity of disease symptoms was marginal, with median values ranging from 0.0 to 0.8 (Table 5), reflecting the ability of $V$. tricorpus isolates to cause minor vascular discoloration in lettuce. Only 2 of the 400 lettuce plants inoculated with $V$. tricorpus alone ever exhibited foliar symptoms typical of Verticillium wilt during the course of these studies. No significant effect of $V$. tricorpus isolate on lettuce fresh foliar and root weight was observed $(P=0.5457$ and 0.5830 , respectively; Table 4) in soildrench-inoculated plants.

In general, lettuce co-inoculated with $V$. dahliae and $V$. tricorpus exhibited reduced disease severity relative to lettuce inoculated with $V$. dahliae alone. A significant effect of $V$. dahliae $\times V$. tricorpus on disease severity was observed when lettuce was inoculated by the soil-drench method $(P<0.0001)$ but not by the root-dip method $(P=0.1629$; Table 4$)$, reflecting the significance of the inoculation method. For example, using the root-dip method, co-inoculation of $V$. tricorpus isolates Ls.432 $(P=0.063)$, Ls.441 $(P=0.014)$, and Ls.443 $(P=0.001)$ with $V$. dahliae isolate Ls. 17 resulted in a significant $57 \%$ reduction in median disease severity relative to lettuce inoculated with Ls.17 alone (Table 5). However, only isolate Ls.443 effectively reduced disease severity when co-inoculated with $V$. dahliae Ls. 16 by the root-dip method (Table 5), albeit with marginal significance $(P=0.083)$. For experiments using the soil-drench method, there were no statistical differences between $V$. tricorpus isolates Ls.432 and Ls.443 $(P=0.662)$ in their effective capacity to reduce disease severity $(P<$ 0.0001 ) by nearly $50 \%$ when co-inoculated with either $V$. dahliae isolate Ls.16 or Ls.17 (Table 5). Although both isolates Ls.432 and Ls.443 reduced disease severity, neither improved fresh foliar or root weight significantly in co-inoculated treatments (Fig. 3).

Pathogenicity on artichoke. Across two independent experiments, the effect of Verticillium isolate on disease severity was significant $(P<0.0001$; Table 6). Artichoke inoculated with $V$. dahliae isolates Cs.80, Cs.312, Cs.413, and Cs.423 exhib- ited significant stunting, reflected in fresh foliar and root weight (Fig. 4), and severe symptoms of Verticillium wilt (Table 7) relative to noninoculated controls. In contrast, V. tricorpus isolates Cs.225, Cs.234, Cs.236, and Cs.456 caused slight but significant $(P=0.002)$ reductions in fresh foliar and root weight (Fig. 4) and Verticillium wilt on artichoke. Mean rankings of disease severity caused by isolates of $V$. dahliae were significantly higher $(P<$ 0.0001 ) than those caused by $V$. tricorpus, with a median disease severity for $V$. dahliae of 5.0, compared with 0.2 for $V$. tricorpus. Although some vascular discoloration was observed in the roots of some artichoke plants inoculated with isolates of $V$. tricorpus, none developed foliar symptoms.

Confirmation of the infection by $V$. tricorpus isolates. After incubation of lettuce and artichoke tissue from inoculated plants on modified NP-10 medium for 10 days in the dark at room temperature, colonies typical of $V$. tricorpus (single-celled conidia on simple conidiophores

Table 6. Statistical analyses of variance (ANOVA) based on the effect of individual isolates of Verticillium dahliae and V. tricorpus or isolate mixtures on the ranked means of Verticillium wilt severity and the means of artichoke measurements across two independent experiments

\begin{tabular}{lccccc}
\hline & \multicolumn{5}{c}{ ANOVA-type statistic (ATS) and $\boldsymbol{F}$ statistic $(\boldsymbol{F})^{\mathbf{a}}$} \\
\cline { 2 - 6 } Isolate effect $^{\mathbf{b}}$ & $\mathbf{d f}_{\text {Num }}$ & $\mathbf{d f}_{\text {Dem }}$ & ATS & $\boldsymbol{F}$ & $\boldsymbol{P}$ value \\
\hline Disease severity & 8 & 39.7 & 38.86 & $\ldots$ & $<0.0001$ \\
Foliar weight & 8 & 40 & $\ldots$ & 12.34 & $<0.0001$ \\
Root weight & 8 & 40 & $\ldots$ & 18.70 & $<0.0001$ \\
Plant height & 8 & 40 & $\ldots$ & 26.80 & $<0.0001$ \\
\hline
\end{tabular}

a Abbreviations: $\mathrm{df}_{\mathrm{Num}}=$ numerator degrees of freedom and $\mathrm{df}_{\mathrm{Dem}}=$ denominator degrees of freedom.

${ }^{\mathrm{b}}$ In the root-dip inoculation method, the roots of 4-week-old artichoke seedlings were dipped in a suspension of approximately $1.0 \times 10^{7}$ conidia/ml for $30 \mathrm{~min}$. Mixed inoculation treatments used a suspension of $1.0 \times 10^{7}$ conidia/ml of each $V$. dahliae and $V$. tricorpus isolate in equal measure. Seedlings were transplanted into 0.5 -liter foam-insulated cups containing a pasteurized sand:potting soil $\operatorname{mix}(3: 1, \mathrm{vol} / \mathrm{vol})$, and sterile water was used in controls.

\section{$\square \mathrm{Vt}-\quad \square \mathrm{VtLs} .432 \quad$ vVtLs.443}

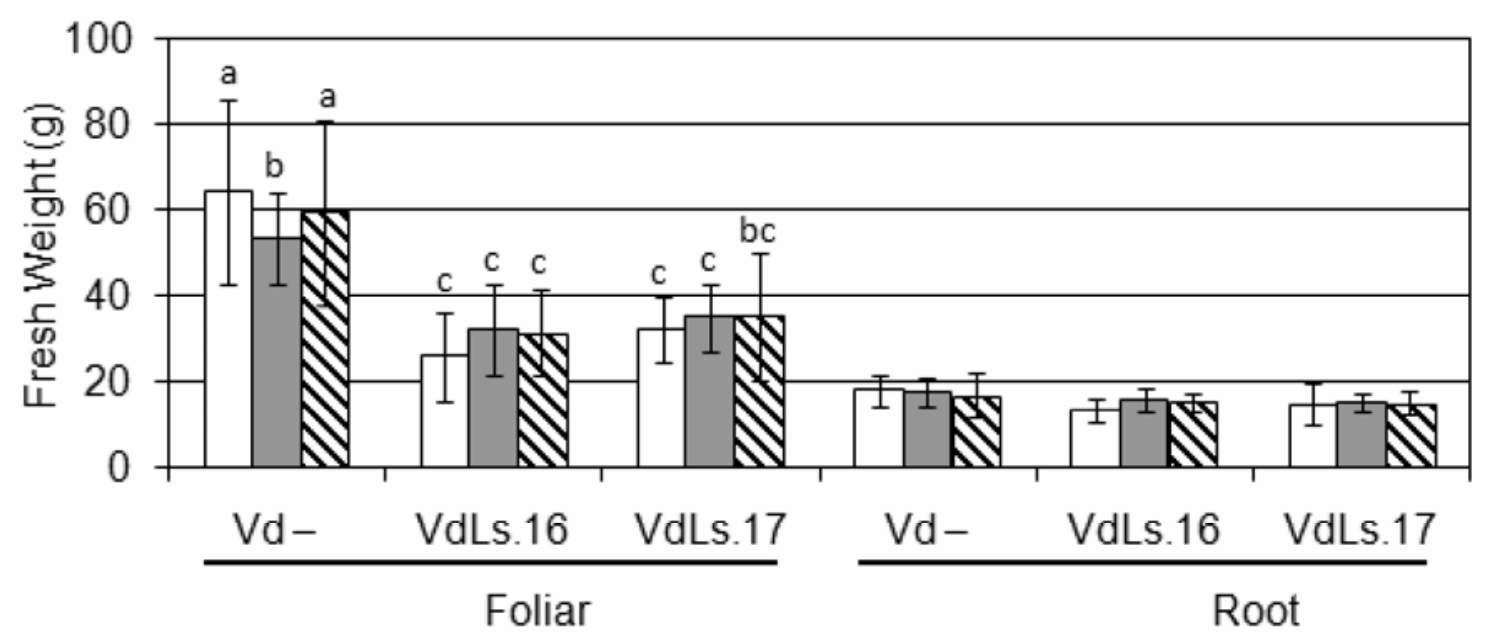

Fig. 3. Effect of individual or mixed isolates of Verticillium dahliae and V. tricorpus on the mean fresh weight of foliar and root tissues of lettuce cv. Salinas 10 to 12 weeks following inoculation. Data represent the least significant (LS) means and standard deviations (bars) from four independent experiments. Differences between foliar weight LS means with the same letters are not statistically significant according to Tukey-Kramer pairwise comparisons at the 95\% confidence level. Such comparisons were not made for root weight, because the V. dahliae-V. tricorpus interaction was not significant. V. dahliae isolates were applied as a conidial suspension to the soil of 4-week-old seedlings prior to transplant. V. tricorpus isolates were applied 1 week prior to the $V$. dahliae isolate. For $\mathrm{Vd}$ and $\mathrm{Vt}$ treatments, sterile water was substituted for the V. dahliae or V. tricorpus isolate, respectively. 
arranged in a verticillate manner along the axis of the hyphae microsclerotia) were observed, regardless of the presence of vascular discoloration. Yellow-orange pigment was observed in colonies formed by segments of tissue from plants inoculated with Ls.183, Ls.442, Cs.225, Cs.234, Cs.236, and Cs.456. V. tricorpus was recovered from 92 to $100 \%$ of inoculated plants. For both lettuce and artichoke plants, either inoculated with $V$. tricorpus alone or in combination with $V$. dahliae, spore-PCR produced the 337-bp diagnostic fragment using the $V$. tricorpus-specific primers and conidia produced by their root tissue samples as a template (Fig. 5 and data not shown). No PCR product was detected from root tissue samples inoculated with isolates of $V$. dahliae alone.

\section{DISCUSSION}

Nine Verticillium isolates (Ls.183, Ls.432, Ls.441, Ls.442, Ls.443, Cs.225, Cs.234, Cs.236, and Cs.456) from lettuce and artichoke were classed as a group distinct from other phytopathogenic Verticillium spp. in a recent study (24). The nine isolates produced dark mycelia, chlamydospores, and microsclerotia and exhibited a pattern of growth across varying temperatures characteristic of $V$. tricorpus isolates $(11,13,27)$. PCR amplification of the nine isolates with primers specific to $V$. tricorpus produced a 377-bp diagnostic fragment. Based on the morphological data and the diagnostic PCR, the nine isolates were identified as $V$. tricorpus. To our knowledge, this is the first reported characterization of $V$. tricorpus isolates from lettuce and artichoke.

Some of the $V$. tricorpus isolates included in this study grew more slowly and produced smaller microsclerotia and fewer
Table 7. Median (Med), mean rankings $(R)$, and relative marginal effects (RME) calculated for the severity of Verticillium wilt on artichoke caused by isolates of Verticillium dahliae and $V$. tricorpus

\begin{tabular}{lccc}
\hline Treatment $^{\mathbf{a}}$ & Med & $\boldsymbol{R}^{\mathbf{b}}$ & $\mathbf{R M E}^{\mathbf{c}}$ \\
\hline K & 0.0 & 8.5 & $0.15(0.11-0.20)$ \\
Cs.312 & 5.0 & 44.5 & $0.81(0.78-0.85)$ \\
Cs.413 & 5.0 & 40.5 & $0.74(0.66-0.81)$ \\
Cs.423 & 5.0 & 42.4 & $0.78(0.70-0.83)$ \\
Cs.80 & 5.0 & 42.6 & $0.78(0.71-0.83)$ \\
Cs.225 & 0.5 & 22.8 & $0.41(0.32-0.51)$ \\
Cs.234 & 0.3 & 17.6 & $0.32(0.23-0.42)$ \\
Cs.236 & 0.1 & 14.5 & $0.26(0.18-0.36)$ \\
Cs.456 & 0.0 & 14.1 & $0.25(0.16-0.39)$ \\
\hline
\end{tabular}

${ }^{a}$ Results from two independent experiments using the root-dip inoculation method.

b Mean rankings of Verticillium wilt severity for each isolate treatment. Disease severity was rated using an ordinal scale of 0 to 5 , in which $0=$ no vascular discoloration in the taproot, $1=1$ to $25 \%, 2$ $=26$ to $50 \%, 3=51$ to $75 \%$, and $4=76$ to $100 \%$ of vascular tissues in the taproot exhibited discoloration in the absence of foliar symptoms, and $5=100 \%$ of vascular tissues exhibited discoloration in the taproot and the presence of foliar symptoms typical of Verticillium wilt.

RME $=[(R-0.5) / \mathrm{N}] ; \mathrm{N}=$ total experimental units in the analysis $(\mathrm{N}=54)$. The $95 \%$ confidence intervals are in parentheses.

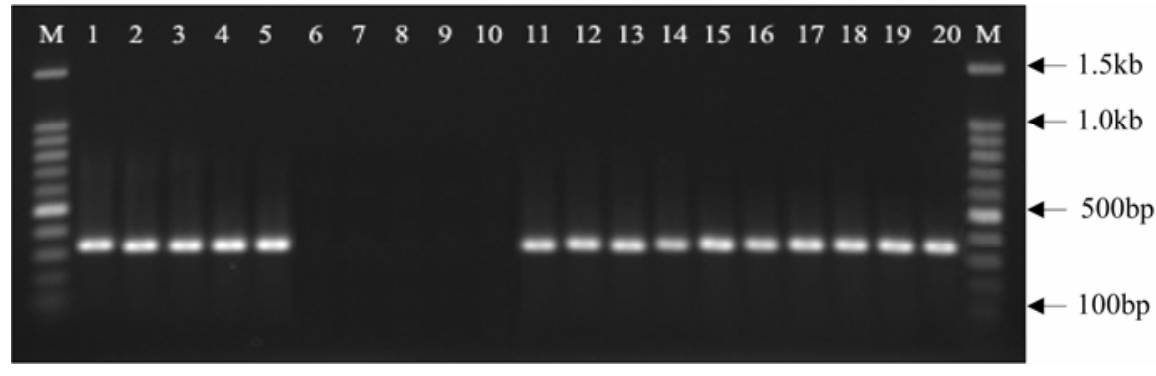

Fig. 5. Confirmation of Verticillium tricorpus on inoculated lettuce roots using V. tricorpus-specific primers in a spore polymerase chain reaction assay. Lanes 1-5 are from lettuce plants inoculated with V. tricorpus isolates Ls.183, Ls.432, Ls.441, Ls.442, and Ls.443, respectively; lanes 6-9 are from lettuce plants inoculated with V. dahliae isolates Ls.1, Ls.14, Ls.16, and Ls.17, respectively; lane 10 is from noninoculated plants; lanes 11-15 are from plants co-inoculated with $V$. dahliae isolate Ls.16 and V. tricorpus isolates Ls.183, Ls.432, Ls.441, Ls.442, and Ls.443, respectively; and lanes 16-20 are from plants co-inoculated with V. dahlia isolate Ls.17 and V. tricorpus isolates Ls.183, Ls.432, Ls.441, Ls.442, and Ls.443, respectively. PCR products were loaded as follows: $6 \mu \mathrm{l}$ on lanes $1-5$ and $10 \mu \mathrm{l}$ on lanes 6-20. M indicates 100-bp DNA ladder (Promega Corp.).

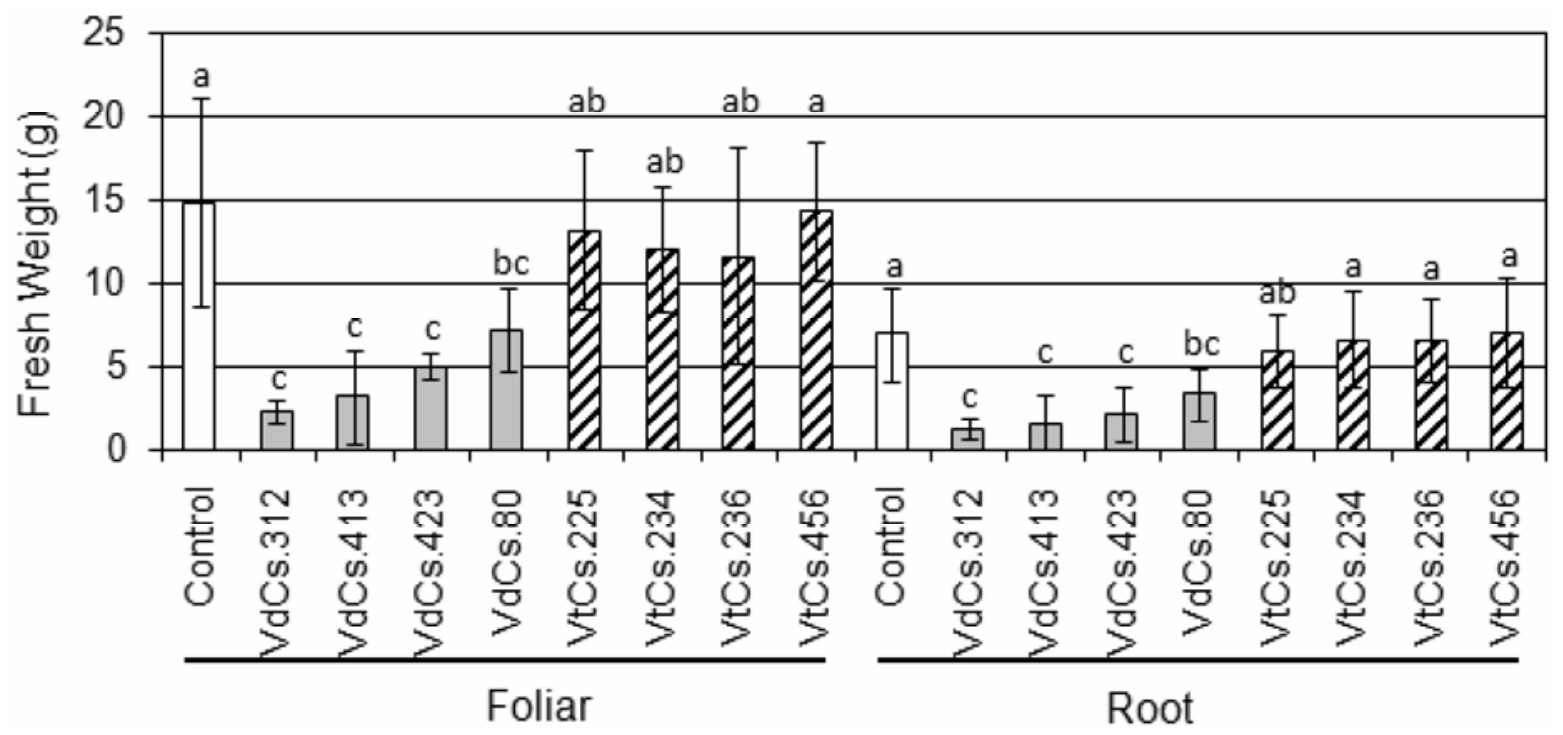

Fig. 4. Effect of individual isolates of Verticillium dahliae and $V$. tricorpus on the mean fresh weight of foliar and root tissues of artichoke 10 to 12 weeks following inoculation. Data represents the least significant (LS) means and standard deviations (bars) from four independent experiments. Differences among foliar weight LS means and among root weight LS means with the same letters are not statistically significant according to Tukey-Kramer pairwise comparisons at the $95 \%$ confidence level. Isolates were applied as a conidial suspension to the soil of 4 -week-old seedlings prior to transplant. Sterile water was used as a control. 
dark resting mycelia and chlamydospores than is typical of this species. These same atypical $V$. tricorpus isolates also failed to produce the characteristic yellow-orange pigment when grown in culture on PDA. These characteristics made it difficult to identify these isolates quickly. The development of PCR primers specific to V. tricorpus should facilitate rapid identification of atypical $V$. tricorpus isolates in future studies.

Using the spore-PCR assay in conjunction with $V$. tricorpus-specific primers aided in the rapid detection of $V$. tricorpus on collected root samples compared with traditional culturing methods or typical PCR methods relying on prior DNA isolation. However, because PCR is performed directly on the spore suspension without any purification, the density of spores in the suspension can have a significant effect on the assay. Having too many or too few spores can result in a failed PCR reaction. For $V$. tricorpus, a concentration of $1.0 \times 10^{5}$ to $1.0 \times 10^{6}$ conidia per $25-\mu$ reaction volume provided adequate amplification.

Regardless of the severity of symptoms, the recovery of $V$. tricorpus from inoculated lettuce and artichoke was very high. The spore-PCR assay consistently produced the 337-bp diagnostic band from all samples from plants inoculated with $V$. tricorpus isolates. These results are consistent with previous reports of high recovery of V. tricorpus from hosts such as eggplant, snapdragon, tobacco, lupin, cotton, and potato $(7,10,11,18,29)$. MacGarvie and Hide (20) estimated that V. tricorpus occurred in $72.5 \%$ of the seed-potato stocks after examining 225 stocks. In field experiments, recovery of $V$. tricorpus from lettuce seedlings was nearly $100 \%$ during the first few weeks following emergence (G. E. Vallad, unpublished data). These results suggest that $V$. tricorpus can readily colonize plant roots. However, significant differences were observed in the pathogenicity of $V$. tricorpus isolates toward lettuce and artichoke. The majority of the $V$. tricorpus isolates used in this study could be considered weakly pathogenic, causing only mild (medium disease severity $<1$ ) but statistically significant symptoms relative to noninoculated lettuce and artichoke plants. This is in agreement with others $(2,13,23)$ who regard V. tricorpus as mildly or weakly pathogenic to hosts.

Verticillium spp. cause specific responses such as early flowering and dying on susceptible hosts (32). Although early flowering of lettuce and premature death of artichoke was quite common among plants inoculated by $V$. dahliae, none of these symptoms occurred on plants inoculated with $V$. tricorpus isolates used in these studies. Of the 400 lettuce plants inoculated with $V$. tricorpus over the course of these studies, only 2 (inoculated with isolates Ls.432 and Ls.441) exhibited foliar symptoms of Verticillium wilt. In Tunisia (15), it was reported that V. tricorpus isolates were highly virulent on eggplant, tomato, and potato, with inoculated plants exhibiting typical foliar wilt symptoms and stunting. A reduction in soybean yield linked to $V$. nigrescens without apparent disease symptoms (33) also was reported. Under greenhouse conditions, the fresh foliar and root weights of plants inoculated with $V$. tricorpus were consistently less than the uninoculated controls, although this trend was statistically negligible. Whether any $V$. tricorpus isolates exist in California with an increased virulence toward lettuce and artichoke, such as those reported in Tunisia (15), is unclear and should be investigated further, as well as the impact of $V$. tricorpus populations on lettuce and artichoke production.

Lettuce co-inoculated with certain isolate combinations of $V$. dahliae and $V$. tricorpus exhibited significantly reduced symptoms of Verticillium wilt, suggesting that specific $V$. tricorpus isolates may protect lettuce against $V$. dahliae through competitive exclusion or cross-protection. Overall, soil-drench inoculations, where the isolate of $V$. tricorpus was applied a week prior to the isolate of $V$. dahliae, appeared to be more robust at reducing Verticillium wilt symptoms relative to the root-dip inoculation method. This suggests that competition plays a major role, because the earlier introduction period would give $V$. tricorpus more time to colonize the root surface, including potential infection sites. However, there was evidence of specificity between certain isolates of $V$. dahliae and $V$. tricorpus. In the root-dip inoculation experiments, V. tricorpus isolates Ls.443, Ls.441, and Ls.432 effectively reduced disease severity against $V$. dahliae isolate Ls.17 but not Ls.16. Of the $V$. tricorpus isolates characterized here, Ls.443 consistently reduced symptoms of Verticillium wilt when co-inoculated with isolates of $V$. dahliae independent of the inoculation method.

Melouk and Horner (21) described cross-protection of peppermint and spearmint against $V$. dahliae when previously inoculated with a related isolate of $V$. nigrescens. More recent reports found $V$. tricorpus to be associated with Verticillium wilt suppression in potato fields (6). Thus, some $V$. tricorpus isolates may act as potential suppressors of Verticillium wilt. So far, however, little is known of the mechanism by which $V$. tricorpus interferes with the more virulent $V$. dahliae. More work is needed to further understand how isolates of V. tricorpus and V. dahliae colonize and interact on a common host over time. Future co-inoculation studies using transformed isolates of $V$. dahliae and V. tricorpus expressing different fluorescent protein color variants may shed light on this topic.
ACKNOWLEDGMENTS

We thank M. Bari for kindly providing artichoke seed for pathogenicity test and B. M. Wu for the suggestions on revising the manuscript.

\section{LITERATURE CITED}

1. Al-Samarrai, T. H., and Schmid, J. 2000. A simple method for extraction of fungal genomic DNA. Lett. Appl. Microbiol. 30:53-56.

2. Barbara, D. J., and Clewes, E. 2003. Plant pathogenic Verticillium species: how many of them are there? Mol. Plant Pathol. 4:297-305.

3. Bhat, R. G., Subbarao, K. V., and Bari, M. A. 1999. First report of Verticillium dahliae causing artichoke wilt in California. Plant Dis. 83:782

4. Bhat, R. G., and Subbarao, K. V. 1999. Host range specificity in Verticillium dahliae. Phytopathology 89:1218-1225.

5. Brunner, E., Domhof, S., and Langer, F. 2002. Nonparametric analysis of Longitudinal Data in Factorial Experiments. John Wiley \& Sons, New York.

6. Davis, J. R., Sorensen, L. H., and Schneider, A. T. 1993. Investigation of the role of Verticillium tricorpus in the biosuppression of Verticillium wilt of potato. (Abstr.) Am. Potato J. 70:806.

7. Ebihara, Y., Nagao, H., Uematsu, S., Moriwaki, J., and Kimishima, E. 2003. First report of Verticillium tricorpus isolated from potato tubers in Japan. Mycoscience 44:481-488.

8. Fradin, E. F., and Thomma, B. P. H. J. 2006. Physiology and molecular aspects of Verticillium wilt diseases caused by $V$. dahliae and $V$. albo-atrum. Mol. Plant Pathol. 7:71-86.

9. Goud, J. K., Termorshuizen, A. J., and Gams, W. 2003. Morphology of Verticillium dahliae and $V$. tricorpus on semi-selective media used for the detection of $V$. dahliae in soil. Mycol. Res. 107:822-830.

10. Huisman, O. C. 1988. Seasonal colonization of roots of field-grown cotton by Verticillium dahliae and $V$. tricorpus. Phytopathology 78:708-716.

11. Isaac, I. 1953. A further comparative study of pathogenic isolates of Verticillium: $V$ nubilum Pethybr and $V$ tricorpus sp nov. Trans. Br. Mycol. Soc. 36:180-195.

12. Isaac, I. 1956. Some soil factors affecting Verticillium wilt of Antirrhinum. Ann Appl. Biol. 44:105-112.

13. Isaac, I. 1967. Speciation in Verticillium. Annu. Rev. Phytopathol. 5:201-222.

14. Isaac, I., and Harrison, J. A. C. 1968. The symptoms and causal agents of early dying disease (Verticillium wilt) of potato. Ann. Appl. Biol. 61:231-244.

15. Jabnoun-khiareddine, H., Daami-Remadi, M. and EI Mahjoub, M. 2005. Emergence en Tunisie de nouveaux pathotypes de Verticillium tricorpus pouvant attaquer la tomate, l'aubergine et la pomme de terre. Bull. OEPP/EPPO Bull. 35:497-503.

16. Kabir, Z., Bhat, R. G., and Subbarao, K. V. 2004. Comparison of media for recovery of Verticillium dahliae from soil. Plant Dis. 88:49-55.

17. Koike, S. T., Subbarao, K. V., Davis, R. M., Gordon, T. R., and Hubbard, J. C. 1994. Verticillium wilt of cauliflower in California. Plant Dis. 78:1116-1121.

18. Korolev, N., and Katan, T. 1999. Vegetative compatibility grouping in Verticillium nigrescens and V. tricorpus. Mycol. Res. 103:65-76.

19. Li, K.-N., Rouse, D. I., and German, T. L. 1994. PCR primers that allow intergeneric differentiation of Ascomycetes and their application to Verticillium spp. Appl. Environ. Microbiol. 60:4324-4331.

20. MacGarvie, Q. D., and Hide, G. A. 1966 Verticillium species from potato seed stocks in Britain in 1965. Plant Pathol. 15:72-75.

21. Melouk, H. A., and Horner, C. E. 1975. Cross 
protection in mint by Verticillium nigrescens against $V$. dahliae. Phytopathology 65:767769.

22. Moukhamedov, R., Hu, X., Nazar, R. N., and Robb, J. 1994. Use of polymerase chain reaction-amplified ribosomal intergenic sequences for the diagnosis of Verticillium tricorpus. Phytopathology 84:256-259.

23. Pegg, G. F., and Brady, B. L. 2002. Verticillium Wilts. CABI Publishing, New York.

24. Qin, Q.-M., Vallad, G. E., Wu, B. M., and Subbarao, K. V. 2006. Phylogenetic analyses of phytopathogenic isolates of Verticillium. Phytopathology 96:582-592.

25. Sambrook, J., Fritsch, E. F., Maniatis, T. 1989. Molecular Cloning: A Laboratory Manual, 2nd ed. Cold Spring Harbor Laboratory Press, Cold
Spring Harbor, NY.

26. Shah, D. A., and Madden, L. V. 2004. Nonparametric analysis of ordinal data in designed factorial experiments. Phytopathology 94:3343.

27. Smith, H. C. 1965 . The morphology of Verticillium albo-atrum, $V$. dahliae and V. tricorpus. N. Z. J. Agric. Res. 8:450-478.

28. Subbarao, K. V., Hubbard, J. C., Greathead, A. S., and Spencer, G. A. 1997. Verticillium wilt. Pages 26-27 in: Compendium of Lettuce Disease. R. M. Davis, K. V. Subbarao, R. N. Raid, and E. A. Kurtz, eds. The American Phytopathological Society, St. Paul, MN.

29. Skotland, C. B. 1971. Pathogenic and nonpathogenic Verticillium species from south central Washington. Phytopathology 61:
434-436.

30. Thanassoulopoulos, C. C., and Giapanoglou, E. 1994. Two new and unusual dry rot of stored potatoes in Greece. Plant Dis. 78:924

31. Uys, M. D. R., Rong, I. H., and Holz, G. 1997. Verticillium tricorpus associated with wilt of tomato in South Africa. Afr. Plant Prot. 3:7375 .

32. Veronese, P., Narasimhan, M. L., Stevenson, R. A., Zhu, J.-K., Weller, S. C., Subbarao, K. V., and Bressan, R. A. 2003. Identification of a locus controlling Verticillium dahliae symptom response in Arabidopsis thaliana. Plant J. 35:574-587.

33. Vesper, S. J., Turner, J. T., and Phillips, D. V. 1983. Incidence of Verticillium nigrescens in soybeans. Phytopathology 73:1338-1341. 